\title{
Association of Micro Albuminuria With Diastolic Function in Obese Normotensive No Diabetic Individuals
}

\author{
Farzanehsadat Minoo $^{1, *}$; Mitra Mahdavi Mazdeh ${ }^{2}$; Mohamad Reza Abasi ${ }^{1}$ \\ ${ }^{1}$ Nephrology Research Center, Tehran University of Medical Sciences, Tehran, IR Iran \\ ${ }^{2}$ Iranian Tissue Bank and Research Center, Tehran University of Medical Sciences, Tehran, IR Iran \\ ${ }^{*}$ Corresponding author: Farzanehsadat Minoo, Nephrology Research Center, Tehran University of Medical Sciences, Tehran, Iran. Tel: +98-2166581568, Fax: +98-2166581568, E-mail: \\ fs-minoo@sina.tums.ac.ir
}

Received: July 28, 2014; Revised: August 11, 2014; Accepted: August 16, 2014

Background: Recently, an association between impaired diastolic function and increased urinary albumin excretion has been hypothesized.

Objectives: We tried to assess the association between diastolic function and micro albuminuria in normotensive no diabetic obese individuals.

Patients and Methods: This cross-sectional study was conducted on 186 consecutive obese normotensive no diabetic individuals who were older than 18 years and attended the outpatient health clinic at the Tehran Municipality in 2011. Systolic and diastolic blood pressures were measured using a standard mercury sphygmomanometer. Micro albuminuria was defined as abnormal urinary albumin to creatinine ratio (UACR) between 30 and $300 \mathrm{mg} / \mathrm{g} / \mathrm{d}$.

Results: An adverse significant linear correlations was found between the UACR measurement and diastolic function $(\mathrm{r}=-0.184$ and $\mathrm{P}=$ 0.012); however, this correlation was insignificant for systolic function $(r=0.007$ and $P=0.926)$. Multivariable linear regression analysis showed that UACR index had a significant reverse correlation with diastolic function (Standardized Beta $=-0.218$ and $\mathrm{P}=0.006$ ).

Conclusions: Our study obtained some evidences on the association of micro albuminuria with diastolic dysfunction in obese normotensive no diabetic individuals. Nonetheless, more assessment is necessary for obtaining a causal relationship between micro albuminuria and diastolic dysfunction.

Keywords:Blood Pressure; Body Mass Index; Micro Albuminuria; Obesity

\section{Background}

Some clinical studies have identified an alteration of cardiac diastolic function in healthy obese patients. It has been demonstrated that obesity could be associated with increased left ventricular (LV) end-diastolic pressure, which suggests an association between obesity and diastolic dysfunction.

Albuminuria is a morbidity of obesity. These two abnormalities can be observe in obesity and can contribute to the prevalence of heart failure and chronic kidney disease in obese patients (1). Nevertheless, we do not know whether these two factors are definitely correlated. If there is any correlation between two factors, physicians should assess second factor when they found first. Micro albuminuria and impaired diastolic function in addition to ventricular remodeling and elevated filling pressures can contribute to the prevalence of heart failure in obese patients (1). These early subclinical abnormalities in LV structure and function might have important implications for explaining the diastolic myocardial dysfunction in obese individuals $(2,3)$. Thus, weight control might reduce these adverse abnormalities such as LV remodeling and preserve LV function (4).
Hypertension (HTN) and diabetes are independently associated with impaired LV diastolic function (5). Several reports have shown that LV diastolic dysfunction is present in patients with diabetes or HTN, even in the absence of the alterations of ventricular systolic function (6-9). However, some evidences indicated diastolic dysfunction with unknown processes in normotensive or non-diabetic subjects $(10,11)$.

Micro proteinuria is a powerful and independent risk factor for cardiovascular morbidity and mortality in diabetic and no diabetic individuals. Body mass index (BMI) has been shown to be significantly correlated with albumin excretion rate and kidney volume and therefore, severe obesity is associated with increased renal plasma flow and glomerular filtration rate (12). In addition, obesity-related glomerular hyper filtration ameliorates after weight loss and the improvement in hyper filtration can prevent the development of overt obesity-related glomerulopathy (13). Some investigators revealed that an elevated albumin excretion rate was associated with an increased diastolic dysfunction $(14-16)$. 


\section{Objectives}

We aimed to assess the association between diastolic function and appearance of micro albuminuria in normotensive no diabetic obese individuals.

\section{Patients and Methods}

The current cross-sectional study was conducted on consecutive obese individuals (BMI $\geq 30 \mathrm{~kg} / \mathrm{m}^{2}$ ) who were older than 18 years and attended the outpatient health clinic at the Tehran Municipality in 2011. Exclusion criteria were history of diabetes mellitus, HTN, hyperlipidemia, cigarette smoking, any renal or liver disorders, urinary tract infections, or menstruation phase of the menstrual cycle. The study was conducted after taking the required ethical clearance from the Ethic Review Board and all patients signed an informed consent.

Baseline data were collected through face-to-face interview and included demographic characteristics and educational level. Weight was measured using a balance standard scale and standing height was measured using a single tape measure. Blood pressure was measured using a standard mercury sphygmomanometer. Diastolic and systolic functions were assessed by a cardiologist through echocardiography. All of the obese patients were referred to one cardiologist and one device was used for echocardiography. Hence, interpersonal and inter-device variability were omitted.

Around $5 \mathrm{~mL}$ of spot urine sample in a sterile container was collected for determination of spot urinary albumin excretion and urinary creatinine. The urinary albumin was measured by immunonephelometric assay, and urinary creatinine was calculated by automated enzymatic assay. Urinary albumin excretion was calculated as urinary albumin to creatinine ratio (UACR). UACR was calculated as milligram of albumin per gram of creatinine. Micro albuminuria was defined as abnormal values of UACR ( $\geq 30 \mathrm{mg} / \mathrm{g}$ ). After ten to 12 hours of overnight fasting, blood samples were collected for estimation of fasting blood sugar (FBS) and serum creatinine. FBS was assayed by glucose oxidase assay kit (Pars Azmoon Ltd. Iran) and serum creatinine was measured by automated enzymatic assay.

Results were reported as mean \pm standard deviation (SD) for the quantitative variables and percentages for the categorical variables. The groups were compared using the Student's t test and the Chi square test (or Fisher's exact test if required) for the continuous and categorical variables, respectively. Correlations between the quantitative variables were examined by the Pearson's correlation test. Predictors that exhibited a statistically significant association with micro albuminuria were taken for multivariate logistic regression analysis to investigate their independence as predictors. Odds ratio (OR) and $95 \%$ confidence intervals ( $95 \% \mathrm{CI}$ ) were calculated. This study was done with the power of $80 \%$. P values $<0.05$ were considered statistically significant. All the statisti- cal analyses were performed using SPSS 13.0 (SPSS Inc., Chicago, IL, USA) and SAS 9.1 for Windows (SAS Institute Inc., Cary, NC, USA).

\section{Results}

The baseline data of the patients are summarized in Table 1. A total of 186 normotensive no diabetic obese individuals were enrolled. The mean age of the subjects was 39.4 years (range, 19-66) with the majority of them being female. The mean BMI was $32.8 \mathrm{~kg} / \mathrm{m}^{2}$ and among them, $17.7 \%$ had severe obesity (BMI $\geq 35 \mathrm{~kg} / \mathrm{m}^{2}$ ). On the first admission day, systolic and diastolic blood pressures were controlled in the normal ranges in all study population. No significant difference in demographic characteristics was found between those with or without micro albuminuria. There were also no significant differences in the baseline laboratory indices including FBS as well as serum creatinine level between two groups.

In univariate regression analysis, a significant correlation between UACR and diastolic function, male sex, age, BMI, serum creatinine, and FBS $(\mathrm{P}<0.05)$ was found. However, the correlation between UACR and weight or educational level was insignificant $(P>0.05)$. Therefore, we used multivariate regression analysis for UACR and diastolic function, male sex, age, BMI, creatinine, and FBS to investigate their independence as predictor (Table 2) and only a significant correlation between UACR and diastolic function was found; hence, so diastolic function was independent factor.

A reverse significant linear correlations was found between the UACR and diastolic function $(r=-0.184$ and $\mathrm{P}=$ 0.012 ), while the correlation between UACR and systolic function was insignificant $(r=0.007$ and $P=0.926)$. Multivariable linear regression analysis (Table 2 ) showed that the UACR had a significant reverse correlation with diastolic function (Standardized Beta $=-0.218$ and $\mathrm{P}=0.006$ ).

\begin{tabular}{|c|c|c|c|c|}
\hline Characteristics & $\begin{array}{l}\text { Total } \\
(n=186)\end{array}$ & $\begin{array}{l}\operatorname{UACR}(+) \\
(\mathbf{n}=\mathbf{2 2})\end{array}$ & $\begin{array}{l}\operatorname{UACR}(-) \\
(\mathbf{n}=\mathbf{1 6 4})\end{array}$ & P value \\
\hline Gender, Male & $11(5.9)$ & $11(6.8)$ & $0(0.0)$ & 0.367 \\
\hline Age, $y$ & $39.4 / 10.7$ & $39.2 / 9.2$ & $39.4 / 10.9$ & 0.921 \\
\hline Weight, kg & $86.5 / 8.2$ & $87.9 / 8.6$ & $86.3 / 8.1$ & 0.426 \\
\hline $\begin{array}{l}\text { Body mass index, } \\
\mathrm{kg} / \mathrm{m}^{2}\end{array}$ & $32.8 / 2.6$ & $33.8 / 3.3$ & $32.7 / 2.5$ & 0.075 \\
\hline Education level & & & & 0.654 \\
\hline Primary & $80(43.7)$ & $8(36.4)$ & $72(44.7)$ & \\
\hline Secondary & $75(41.0)$ & $11(50.0)$ & $64(39.8)$ & \\
\hline College degree & $28(15.3)$ & $3(13.6)$ & $25(15.5)$ & \\
\hline $\begin{array}{l}\text { Fasting blood } \\
\text { sugar, g/dL }\end{array}$ & $96.0 / 23.9$ & $100.2 / 23.7$ & $95.4 / 24.0$ & 0.385 \\
\hline $\begin{array}{l}\text { Serum creatinine, } \\
\mathrm{mg} / \mathrm{dL}\end{array}$ & $0.83 / 0.11$ & $0.82 / 0.11$ & $0.83 / 0.11$ & 0.565 \\
\hline
\end{tabular}


Minoo F et al.

Table 2. Association Between Microalbuminuria and Diastolic Function in the Presence of Other Variables as Probable Cofounders

\begin{tabular}{|c|c|c|c|}
\hline Variable & Standardized Beta & T Score & Pvalue \\
\hline Diastolic Function & -0.218 & -2.777 & 0.006 \\
\hline Male, Gender & 0.024 & 0.311 & 0.756 \\
\hline Age & 0.09 & 0.128 & 0.898 \\
\hline Body Mass Index & -0.004 & -0.054 & 0.957 \\
\hline Serum Creatinine & -0.030 & -0.388 & 0.699 \\
\hline Fasting Blood Sugar & 0.086 & 1.105 & 0.271 \\
\hline
\end{tabular}

\section{Discussion}

We observed a reverse association between diastolic dysfunction and UACR, which indicated a negative association between micro albuminuria and diastolic function. Some observations have shown that the more relevant impairment in diastolic function in patients with micro albuminuria could be related to the increased LV mass index with concentric remodeling (14). On the other hand, LV mass and LV wall thickness can be significantly increased and LV relaxation might be impaired in micro albuminuria (14). Picca et al. revealed that an elevated urinary albumin excretion rate was associated with an increased LV mass index, a higher prevalence of a concentric LV hypertrophy pattern, a depressed mid-wall systolic performance, and a markedly impaired diastolic function $(15,16)$. These evidences can confirm potential association between micro albuminuria and diastolic dysfunction. According to this fact that the diastolic function can be directly dependent to some pointed structural and functional indices, these cardiac indices should be assessed in those with the evidences of micro albuminuria, especially those who are suspected to have ventricular diastolic dysfunction.

Our study focused on the presence of micro albuminuria in obese no diabetic normotensive patients; therefore, our finding was adjusted for other potential predisposing factors of micro albuminuria such as HTN and diabetes mellitus. In addition, the role of obesity in the development and progression of diastolic dysfunction has been more highlighted. Some studies revealed that obesity might be more important than micro albuminuria for the prediction and prevention of diastolic dysfunction in no diabetic patients (17). In this context, mediating role of inflammatory processes, particularly in normotensive no diabetic individuals, should not be ignored. It has been demonstrated that inflammatory fibrosis of the myocardium is a major cause of myocardial stiffening, which leads to dysfunction. The higher prevalence of proinflammatory conditions as well as higher levels of inflammatory markers can be often found in those with myocardial stiffening and diastolic dysfunction (18-22). Moreover, in acute inflammation, micro albuminuria might be a result of the endothelial glomerular leak in the kidneys, which is a manifestation of the systemic increases in capillary permeability due to an intense inflammatory onslaught on the endothelium $(23,24)$. Each acute inflammatory condition might lead to endothelial glomerular defects and finally result in micro albuminuria. Thus, the association between impaired diastolic function and micro albuminuria might be mediated by inflammatory processes. Therefore, those who are suspected of concurrent micro albuminuria and diastolic dysfunction, especially those with any evidence of heart failure, should be screened for acute or chronic inflammatory disorders.

In summary, our results obtained some evidences regarding association between micro albuminuria and diastolic dysfunction in obese no diabetic normotensive individuals; however, more assessments are necessary for obtaining the casualty in this association.

\section{Acknowledgements}

This manuscript was the presentation of the subspecialty fellowship thesis and was supported by Tehran University of Medical Sciences. We thank Tehran University of Medical Sciences for their support.

\section{Authors' Contributions}

Study concept and design: Farzanehsadat Minoo, Mitra Mahdavi Mazdeh, and Mohamad Reza Abasi. Analysis and interpretation of data: Mitra Mahdavi Mazdeh, Farzanehsadat Minoo. Drafting of the manuscript and critical revision of the manuscript for important intellectual content: Farzanehsadat Minoo. Statistical analysis: Mitra Mahdavi Mazdeh.

\section{References}

1. Powell BD, Redfield MM, Bybee KA, Freeman WK, Rihal CS. Association of obesity with left ventricular remodeling and diastolic dysfunction in patients without coronary artery disease. Am J Cardiol. 2006;98(1):116-20.

2. Peterson LR, Waggoner AD, Schechtman KB, Meyer T, Gropler RJ, Barzilai B, et al. Alterations in left ventricular structure and function in young healthy obese women: assessment by echocardiography and tissue Doppler imaging. $\mathrm{J}$ Am Coll Cardiol. 2004;43(8):1399-404.

3. Wong CY, O'Moore-Sullivan T, Leano R, Byrne N, Beller E, Marwick TH. Alterations of left ventricular myocardial characteristics associated with obesity. Circulation. 2004;110(19):3081-7.

4. Dorbala S, Crugnale S, Yang D, Di Carli MF. Effect of body mass index on left ventricular cavity size and ejection fraction. Am J Cardiol. 2006;97(5):725-9.

5. Russo C, Jin Z, Homma S, Rundek T, Elkind MS, Sacco RL, et al. Effect of diabetes and hypertension on left ventricular diastolic function in a high-risk population without evidence of heart disease. Eur J Heart Fail. 2010;12(5):454-61.

6. Celentano A, Vaccaro O, Tammaro P, Galderisi M, Crivaro M, Oliviero $\mathrm{M}$, et al. Early abnormalities of cardiac function in noninsulin-dependent diabetes mellitus and impaired glucose tolerance. Am J Cardiol. 1995;76(16):1173-6.

7. Zabalgoitia M, Ismaeil MF, Anderson L, Maklady FA. Prevalence of diastolic dysfunction in normotensive, asymptomatic patients with well-controlled type 2 diabetes mellitus. Am J Cardiol. 2001;87(3):320-3.

8. Boyer JK, Thanigaraj S, Schechtman KB, Perez JE. Prevalence of ven- 
tricular diastolic dysfunction in asymptomatic, normotensive patients with diabetes mellitus. Am J Cardiol. 2004;93(7):870-5

9. Fang ZY, Schull-Meade R, Leano R, Mottram PM, Prins JB, Marwick TH. Screening for heart disease in diabetic subjects. Am Heart $J$ 2005;149(2):349-54.

10. Ojji D, Parsonage W, Dooris M, Adebiyi A, Oladapo O, Adeleye J, et al. Left ventricular diastolic function in normotensive type-2 diabetic subjects. J Natl Med Assoc. 2008;100(9):1066-72.

11. Patil VC, Patil HV, Shah KB, Vasani JD, Shetty P. Diastolic dysfunc tion in asymptomatic type 2 diabetes mellitus with normal systolic function. J Cardiovasc Dis Res. 2011;2(4):213-22.

12. Lokkegaard N, Haupter I, Kristensen TB. Microalbuminuria in obesity. Scand J Urol Nephrol.1992;26(3):275-8.

13. Chagnac A, Weinstein T, Herman M, Hirsh J, Gafter U, Ori Y. The effects of weight loss on renal function in patients with severe obesity. J Am Soc Nephrol. 2003;14(6):1480-6.

14. Guglielmi MD, Pierdomenico SD, Salvatore L, Romano F, Tascione E, Pupillo M, et al. Impaired left ventricular diastolic function and vascular postischemic vasodilation associated with microalbuminuria in IDDM patients. Diabetes Care.1995;18(3):353-60.

15. Picca M, Agozzino F, Pelosi G. Influence of microalbuminuria on left ventricular geometry and function in hypertensive patients with type 2 diabetes mellitus. Ital Heart J. 2003;4(1):48-52.

16. Duman D, Demirtunc R, Karadag B, Karatas M. Association of albuminuria with impaired aortic elasticity and left ventricula diastolic dysfunction in type 2 diabetes. Anadolu Kardiyol Derg.
2008;8(1):10-5.

17. Shemirani H, Khosravi A, Hemmati R, Gharipour M. Body Mass Index or Microalbuminuria, Which One is More Important for the Prediction and Prevention of Diastolic Dysfunction in Nondiabetic Hypertensive Patients? Int J Prev Med. 2012;3(3):211-20.

18. Zile MR, Brutsaert DL. New concepts in diastolic dysfunction and diastolic heart failure: Part II: causal mechanisms and treatment. Circulation. 2002;105(12):1503-8.

19. Ho CY, Lever HM, DeSanctis R, Farver CF, Seidman JG, Seidman CE. Homozygous mutation in cardiac troponin T: implications for hypertrophic cardiomyopathy. Circulation. 2000;102(16):1950-5.

20. van Hoeven $\mathrm{KH}$, Factor SM. A comparison of the pathologica spectrum of hypertensive, diabetic, and hypertensive-diabetic heart disease. Circulation. 1990;82(3):848-55.

21. Kuwahara F, Kai H, Tokuda K, Takeya M, Takeshita A, Egashira K, et al. Hypertensive myocardial fibrosis and diastolic dysfunction: another model of inflammation? Hypertension. 2004;43(4):73945.

22. Albert MA, Torres J, Glynn RJ, Ridker PM. Perspective on selected issues in cardiovascular disease research with a focus on black Americans. Circulation. 2004;110(2):e7-12.

23. Gosling P. Microalbuminuria: a marker of systemic disease. $\mathrm{Br}$ Hosp Med. 1995;54(6):285-90.

24. Dziedzic T, Slowik A, Szczudlik A. Urine albumin excretion in acute ischaemic stroke is related to serum interleukin-6. Clin Chem Lab Med. 2004;42(2):182-5. 